\title{
Slow plasmonic slab waveguide as a superlens for visible light
}

\author{
J. Christensen* and F. Javier García de Abajo ${ }^{\dagger}$ \\ Instituto de Optica, CSIC, Serrano 121, 28006 Madrid, Spain \\ (Received 10 September 2010; published 19 October 2010)
}

\begin{abstract}
We propose a concept of a near-field lens capable of reliably imaging deep-subwavelength objects at a distance of several times the thickness of the lens. Nearly flat guided modes in a high-index dielectric slab are shown to propagate information of evanescent waves emanating from the imaged object, thus providing the degree of detail needed to map fine spatial variations. A practical way of implementing the proposed flat lens is theoretically demonstrated using closely packed metal nanoparticles giving rise to large effective permittivities in the spectral region right to the red of the plasma frequency. We perform rigorous electromagnetic simulations showing that realistic absorption levels in the metal are still compatible with deep-subwavelength spatial resolution.
\end{abstract}

DOI: $10.1103 /$ PhysRevB.82.161103

PACS number(s): 78.20.Ci, 42.30.Wb, 73.20.Mf, 78.66.Bz

Metamaterial-based superlenses have sparked considerable interest in the quest to obtain the finest possible imaging spatial resolution beyond the capabilities of conventional lenses. Abbe's (1873) diffraction, which limits the performance of these lenses to resolve objects below about half the wavelength in size, seemed for a long time to be a major obstacle in achieving this goal, although now it has been circumvented by cunningly contrived designs. However, despite the availability of innovative negative-index metamaterial designs which are needed to make perfect lenses, ${ }^{1}$ intrinsic metal losses and nanofabrication tolerances are seriously averting their realization in the useful range of the visible and near infrared. In this rapid communication we propose a plasmonic strategy to pursuit a metamaterial superlens working for visible light. We demonstrate that by excitation of guided modes inside a slab of high positive index of refraction, a subwavelength object can be captured by a sizeable range of its propagating and evanescent near-field components at a significant distance in the image plane. We show through rigorous electromagnetic simulation how this idea can be implemented using the collective plasmonic response of a film formed by closely packed nanoparticles.

The resolution of an ordinary lens is restricted by the diffraction limit, which only permits obtaining images from propagating waves emitted or scattered by an object to be captured. However, evanescent waves, which cannot travel far away from the object because of their exponentially decaying wave nature, keep all the fine details in the near-field unreachable for the observer. With the emergence of negative-index materials (NIMs) the tide has turned with regards to the evanescent waves which would now experience amplification inside a metamaterial slab containing $\varepsilon=\mu=$ -1 , such that theoretically unlimited lensing can be reached. ${ }^{2}$ Isotropic NIM lenses have been demonstrated this way in the microwave but also in the mid IR and optical regime within the electrostatic limit. $^{3-5}$ Indefinite (anisotropic) media as proposed by Smith and Schurig, ${ }^{6}$ contain hyperbolic dispersion relations that have been realized for nonmagnetic metamaterials with the ability to convert evanescent waves associated to higher spatial harmonics into propagating waves. ${ }^{7}$ Utilizing this technique, onion structures ${ }^{8-11}$ and silver nanowire systems ${ }^{12,13}$ have successfully been implemented for negative permittivity metamaterials with negative refraction. Another imaging scheme comprising parallel wires (the so-called endoscope) possesses the ability of efficiently transferring subwavelength features through the structure when standing waves are excited. ${ }^{14,15}$ When these wires are crossed, they form an effective medium with extreme effective parameter in the microwave. ${ }^{16}$

What all these suggested routes for achieving metamaterial-based superlensing may share in common is the fact that slowing of light at metallic surfaces is taking place. Whether plasmonic, indefinite, or wire media are being employed, resonances in those metamaterials are supported for high values of the parallel momentum corresponding to fine details in the object. Let us try to explore this for a general case of a slow dielectric slab. In the absence of external currents and charges, Ampere's law and Faraday's law can be combined to write Maxwell's equation as

$$
\nabla \times \nabla \times \mathbf{E}-\left[\frac{2 \pi n(\mathbf{r})}{\lambda}\right]^{2} \mathbf{E}=0
$$

where $n(\mathbf{r})$ is the space-dependent index of refraction, $\mathbf{E}(\mathbf{r})$ is the electric field, and $\lambda$ is the free-space wavelength. For $s$-polarized irradiation (TE, with the electric field perpendicular to the $z$-axis) and translational invariance along the $x y$ plane, we define the index of refraction as $n(z)$ across the slab of thickness $T$. For this particular case we use a quantum-mechanical analogy in order to conquer insight into the optical problem. We assimilate our system to a onedimensional potential $V(z)=\left[1-n^{2}(z)\right] \frac{2 \pi^{2}}{\lambda^{2}}$ with the light represented by a particle of unit mass and effective energy $E$ $=\left(1-n_{s}^{2} \sin ^{2} \theta_{s}\right) \frac{2 \pi^{2}}{\lambda^{2}}$, where $\theta_{s}$ is the incidence angle with respect to $z$ in medium $s$ of refraction index $n_{s}$. We take $n_{s}$ and $n_{0}=1$ to be the refractive indices inside and outside the slab, respectively. The slab then defines a potential well. To ensure the particle to be confined inside the well in the form of a total reflected wave, obviously the potential in the barrier must be larger than the particle energy, $V_{0}>E$. Likewise light is trapped in a region of high refractive index or with boundaries of high reflectivity. ${ }^{17}$ This analogy is best explained by taking 


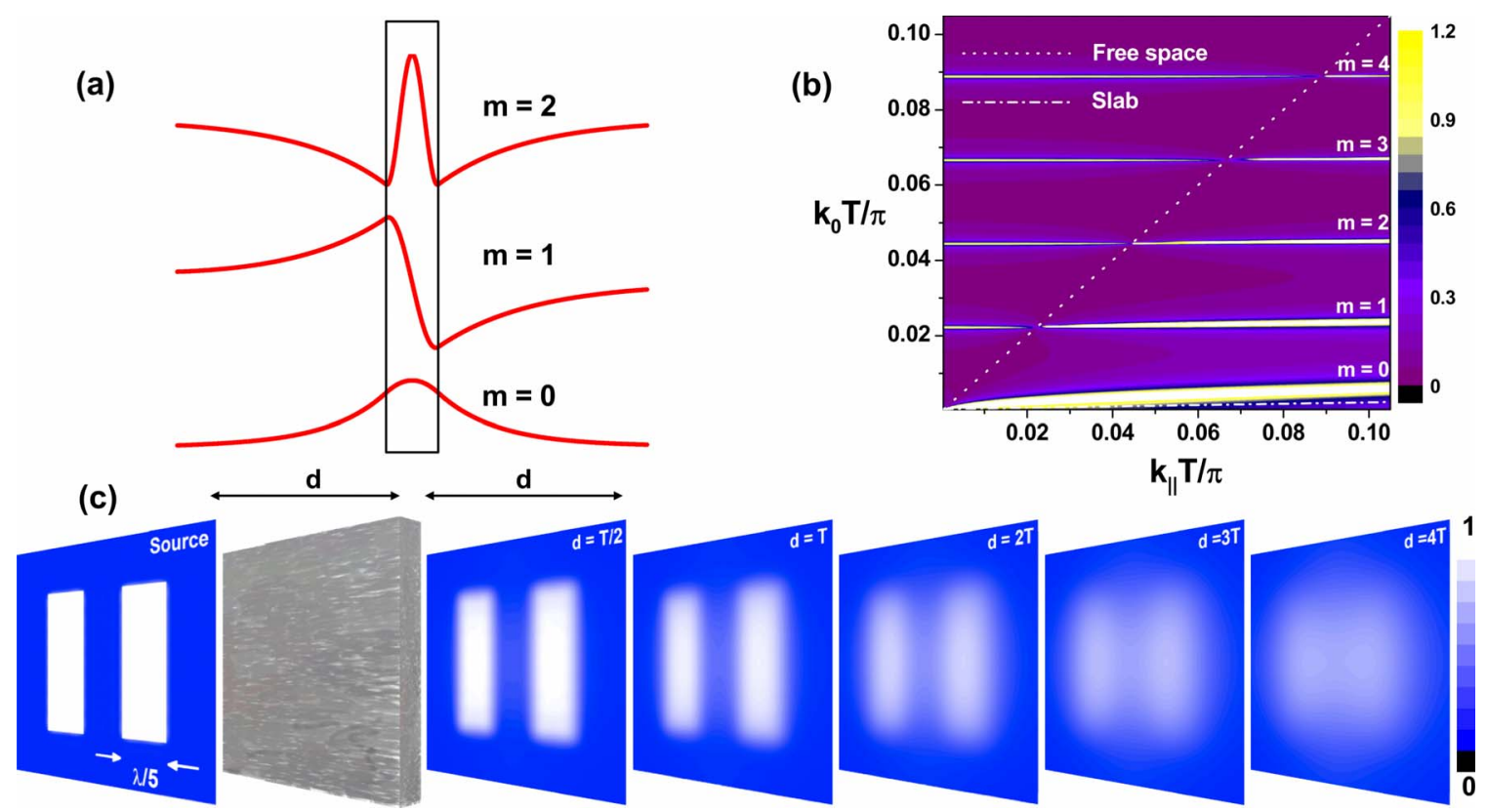

FIG. 1. (Color online) (a) Field amplitudes for $\mathrm{TE}_{0}, \mathrm{TE}_{1}$, and $\mathrm{TE}_{2}$ modes in a dielectric waveguide. (b) Bound and radiating modes: transmission $t$ through a dielectric slab of $n_{s}=45$ as a function of free space and parallel wave vectors, $k_{0}$ and $k_{\|}=k_{x}$, respectively, normalized to the slab thickness $T$. Dotted and dashed-dotted lines are the light cones in free space and inside the slab, respectively. The first five discrete bound modes $(m=0,1,2,3,4)$ are plotted. (c) Imaging configuration: two bars constituting a complex subwavelength source of width $w$ $=\lambda / 5$ emit $s$-polarized light from a distance $d$ to the lens. The object is imaged at a plane located at a distance $d$ away from the slab, such that for all illustrated cases we take $d_{\text {object }}=d_{\text {image }}=d$.

$$
\mathbf{E}(\mathbf{r})=\psi(z) e^{i k_{y} y} \hat{\mathbf{x}}
$$

as an ansatz incident wave and substituting it into Eq. (1). With the parallel momentum $k_{y}=n_{s} \frac{2 \pi}{\lambda} \sin \theta_{s}$ and the identity $\nabla \times \nabla \times \mathbf{E}=-\nabla^{2} \mathbf{E}+\nabla(\nabla \cdot \mathbf{E})$, we obtain the time-independent Schrödinger equation

$$
-\frac{1}{2} \frac{d^{2} \psi(z)}{d z^{2}}+V(z) \psi(z)=E \psi(z) .
$$

This quantum-mechanical finite potential-well problem, which is mimicking the slow light system, is well known in connection with dielectric slab waveguides. The discrete bound states [see the first three guided modes in Fig. 1(a)] are subject to $n_{s}>n_{s} \sin \theta_{s}>n_{0}$, which is the confinement condition. Together with resonant states above the potential barrier, which are unbound just like the radiating modes of a slab above cutoff, propagating and evanescent waves can be efficiently transferred through the slab of thickness $T$. The challenge, however, lies in the uniform transfer of all spatial harmonics with a significant emphasis on the evanescent wave components-ideally in the form of near-field amplification. Thus it is clear that high transmission must be preserved through a medium with little dispersion as has been exemplified in Fig. 1(b) for a dielectric layer of $n_{s}=45$. As can be seen in this figure, a discrete set of flat bands are resonantly excited. When the location of the mode approaches cutoff, most of the field leaks out of the guide. However below cutoff the transmission through the slow slab can exceed unity due to $n_{s} \gg n_{0}$. The overall transmission after multiple-scattering events reduces to

$$
t=\frac{\tau \tau^{\prime} e^{i k_{z}^{\prime} T}}{1-r^{2} e^{2 i k_{z}^{\prime} T}},
$$

which has been employed for these calculations. Here, $\tau, \tau^{\prime}$ and $r$ are the Fresnel transmission and reflection coefficients of the $n_{0} / n_{s}$ interface, whereas $k_{z}^{\prime}=\sqrt{n_{s}^{2} \frac{4 \pi^{2}}{\lambda^{2}}-k_{\|}^{2}}$. Resonant transmission exceeding unity is obtained whenever the resonant condition

$$
-2 \varphi+k_{z}^{\prime} T=m \pi
$$

is fulfilled, where $\varphi=\tan ^{-1} \frac{1}{k_{z}^{\prime}} \sqrt{k_{\|}^{2}-n_{0}^{2} \frac{4 \pi^{2}}{\lambda^{2}}}$ is the GoosHänchen shift. A slow dielectric slab is hosting multiples of those modes as Fig. 1(b) illustrates, and form the basis to amplify evanescent waves. By virtue of the flatness of those curves, Fig. 1(c) shows for $k_{0} T / \pi=0.045$ how a subwavelength object (two $\lambda / 5$ bars) can be imaged down to the finest details. As an important feature within this scheme, we emphasize that the equally chosen distances of the object and image planes from the slab $\left(d_{\text {object }}=d_{\text {image }}=d\right)$ are exceeding those of what is achievable when a NIM or endoscope is being employed. ${ }^{2,14}$ Figure 1(c), showing $\left|\Psi_{\text {out }} / \Psi_{\text {in }}\right|$ as calculated by integrating the product of Eq. (4) with the source in momentum space, demonstrates that imaging is achievable up to $d=3 T$. Next we calculate the transmitted image intensity $\Psi_{\text {out }}$ for various values of the refraction index of the slab $n_{s}$. When varying this value both the flatness and the spectral location of the bound modes change, hence we concentrate the calculation around the $\mathrm{TE}_{2}$ mode for all values of $n_{s}$ but with $\lambda / w$ constant. For the largest values of the index, al- 


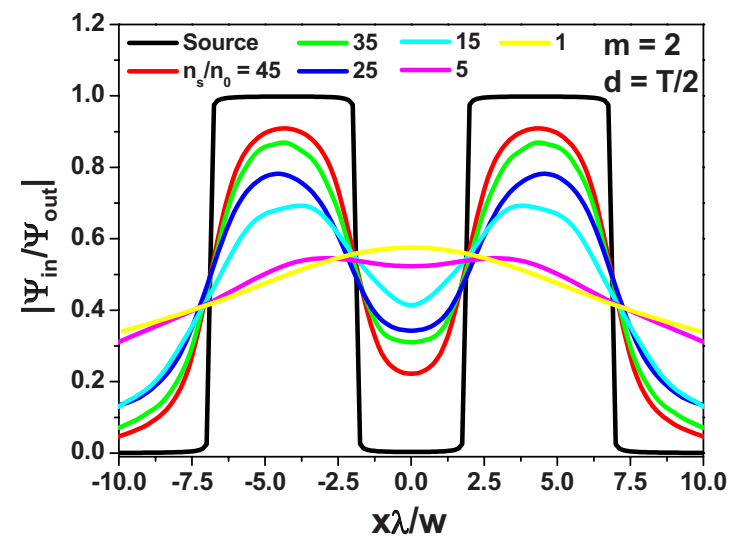

FIG. 2. (Color online) $\left|\Psi_{\text {out }} / \Psi_{\text {in }}\right|$ at the image plane $(d=T / 2)$ for different values of $n_{s}$ in the slab. In all cases the images are plotted for the $\mathrm{TE}_{2}$ mode at a fixed index of refraction corresponding to wavelengths five times larger than the source width $w$.

most flawless objects are imaged in Fig. 2. In contrast, the faster the phase velocity of the slab, the more details are lost and, as expected when $n_{s} \rightarrow 1$, an almost ordinary lens is obtained, incapable to function well below the diffraction limit.

In the following we shall employ a plasmonic metamaterial that can work as a high-index system of low angular sensitivity. Nearly touching metallic nanoparticle arrays behave as artificial dielectric bulk layers of high effective permittivity $\varepsilon_{e f f}$ due to polarization of particle gap-plasmon resonances. ${ }^{18,19}$ We focus on the long-wavelength tail of this Lorentzian resonance because this zone contains a high index of refraction, as seen in Fig. 3(a) for two different filling fractions (ff), ideally suitable for imaging applications. The origin of this high electric response can be traced back to intense field concentration in the gap zone of neighboring spheres. Similar phenomena have been described for slit arrays with localized resonances. ${ }^{20}$ We now wish to use a slab of this metamaterial as a lens whereby we take advantage of the plasmon tunability controlled by the filling fraction. Slab guided modes are supported for nanoparticle structures of slab thickness $T=100 \mathrm{~nm}$, both with $\mathrm{ff}=58 \%$ at $\lambda$ $=590 \mathrm{~nm}\left(n_{\text {eff }}=13.7+i 1.8\right)$ and for $\mathrm{ff}=70 \%$ at $\lambda=825 \mathrm{~nm}$ $\left(n_{\text {eff }}=12.5+i 0.18\right)$. Despite losses in the nanoparticle metamaterial near resonance for the proposed filling fractions, distinguishable subwavelength features can be identified in the image for both orange light, Fig. 3(c), and near-
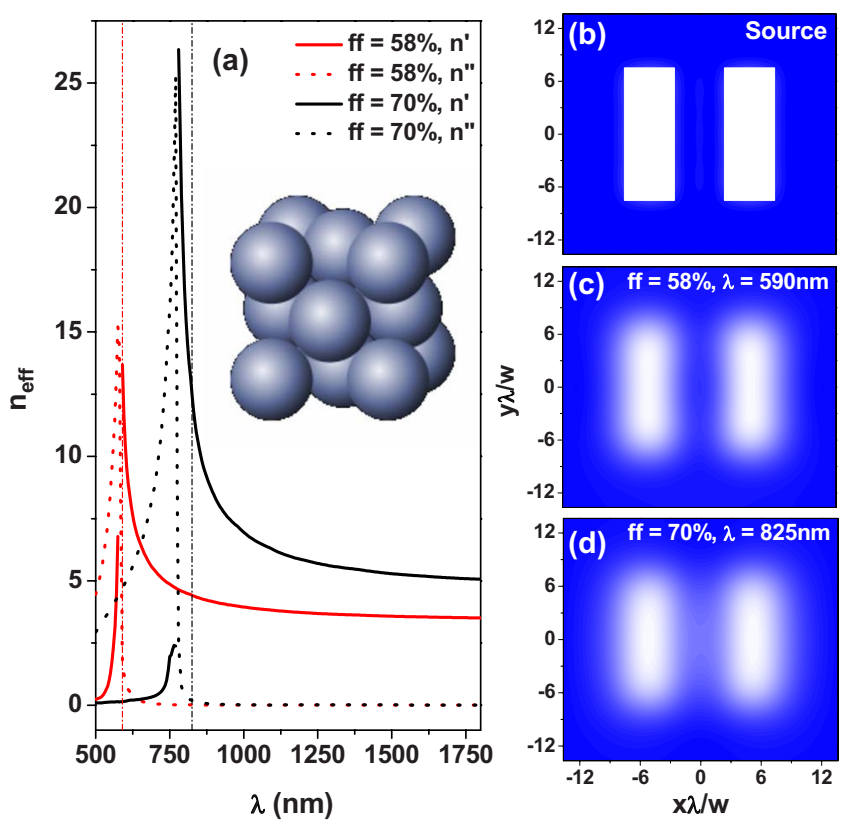

FIG. 3. (Color online) (a) Silver nanoparticle fcc array embedded in silica for two different filling fractions: $58 \%$ and $70 \%$. Calculated effective index of refraction $n_{\text {eff }}=n^{\prime}+i n^{\prime \prime}$, as reported in Ref. 19. $n^{\prime}$, full lines and $n^{\prime \prime}$, dotted lines. (b) Source. (c) $d=T / 2$ with $\left|\Psi_{\max }\right|=0.2$ and (d) $d=T / 2$ with $\left|\Psi_{\max }\right|=0.3$, both normalized to $\left|\Psi_{\text {in }}\right|$. We take $T=100 \mathrm{~nm}$.

infrared radiation, Fig. 3(d). Interestingly the superimposed intensity between the subwavelength bars seem to vanish when losses are high.

Subwavelength imaging based on high-index materials has been demonstrated using plasmonic metamaterials for this concept to work in the visible. Unlike other approaches that transfer subwavelength information to the nearest vicinity of the structure, one could utilize the current system to function at further distances than half the slab thickness. We foresee that this right-handed slow plasmonic lens will be important because it is a technically feasible scheme to implement subwavelength optical applications such as imaging and lithography beyond the diffraction limit.

This work has been supported by the Spanish MICINN (Consolider NanoLight.es). J.C. gratefully acknowledges financial support from the Carlsberg Foundation under Contract No. QUANTONICS 2009-01-0167.

\footnotetext{
*johan.christensen@gmail.com

†.g.deabajo@csic.es

${ }^{1}$ J. Valentine, S. Zhang, T. Zentgraf, E. Ulin-Avila, D. A. Genov,

G. Bartal, and X. Zhang, Nature (London) 455, 376 (2008).

${ }^{2}$ J. B. Pendry, Phys. Rev. Lett. 85, 3966 (2000).

${ }^{3}$ A. A. Houck, J. B. Brock, and I. L. Chuang, Phys. Rev. Lett. 90, 137401 (2003).

${ }^{4}$ N. Fang, H. Lee, C. Sung, and X. Zhang, Science 308, 534 (2005).
}

${ }^{5}$ T. Taubner, D. Korobkin, Y. Urzhumov, G. Shvets, and R. Hillenbrand, Science 313, 1595 (2006).

${ }^{6}$ D. R. Smith and D. Schurig, Phys. Rev. Lett. 90, 077405 (2003).

${ }^{7}$ Z. Jacob, L. V. Alekseyev, and E. Narimanov, Opt. Express 14, 8247 (2006).

${ }^{8}$ J. B. Pendry and S. A. Ramakrishna, J. Phys.: Condens. Matter 14, 8463 (2002).

${ }^{9}$ A. Salandrino and N. Engheta, Phys. Rev. B 74, 075103 (2006).

${ }^{10}$ Z. Liu, H. Lee, Y. Xiong, C. Sun, and X. Zhang, Science 315, 
1686 (2007).

${ }^{11}$ I. I. Smolyaninov, Y.-J. Hung, and C. C. Davis, Science 315, 1699 (2007).

${ }^{12}$ J. Yao, Z. Liu, Y. Liu, Y. Wang, C. Sun, G. Bartal, A. Stacy, and X. Zhang, Science 321, 930 (2008).

${ }^{13}$ A. Fang, T. Koschny, and C. M. Soukoulis, Phys. Rev. B 79, 245127 (2009).

${ }^{14}$ P. A. Belov and Y. Hao, Phys. Rev. B 73, 113110 (2006).

${ }^{15}$ P. A. Belov, Y. Hao, and S. Sudhakaran, Phys. Rev. B 73, 033108 (2006).
${ }^{16}$ M. G. Silveirinha, C. R. Medeiros, C. A. Fernandes, and J. R. Costa, Phys. Rev. B 81, 033101 (2010).

${ }^{17}$ H. A. Haus and D. A. B. Miller, IEEE J. Quantum Electron. 22, 310 (1986).

${ }^{18}$ D. R. McKenzie and R. C. McPhedran, Nature (London) 265, 128 (1977).

${ }^{19}$ R. Sainidou and F. J. García de Abajo, Opt. Express 16, 4499 (2008).

${ }^{20}$ J. T. Shen, P. B. Catrysse, and S. Fan, Phys. Rev. Lett. 94, 197401 (2005). 